\title{
Emergency obstetric care, essentialities for prevention of maternal/neonatal mortality/morbidity
}

\begin{abstract}
Introduction: Many disorders during pregnancy, labour can neither be predicted nor prevented. It is essential that there is timely detection of such disorders during pregnancy, labour, postpartum to prevent, severe morbidity, mortality by appropriate, emergency obstetric care.

Present status: During last 3 decades, since International Safe Motherhood Conference, it has become evident that for quick impact on maternal mortality, severe morbidity, it is essential that obstetric emergencies are prevented, if not prevented, detected, managed timely and appropriately. This requires knowledge, skill, infrastructure, material and policies. Many disorders kill women but actions needed are few, homeostasis, antibiotics, appropriate surgery and other symptomatic therapy. Awareness of this is essential for all those dealing with maternal health. This needs knowledge, skill of caesarean section and also knowledge of judicious use of CS because although cesarean birth provides relatively safe outcome, it increases morbidity/mortality. Knowledge, skill to do CS, while part of essentialities of emergency services, is not panacea of prevention of morbidity, mortality, as deaths are not only because CS is not available, but also because hypertension, sepsis, postpartum hemorrhage and other obstetric emergencies are not prevented, not diagnosed in time, not treated appropriately and timely.
\end{abstract}

Possibilities: Focus on interventions that are designed to avert deaths during pregnancy, around delivery is essential. Timely appropriate referral is essential with skilled specialists / non-specialists knowing their job, with knowledge, skills with comprehensive understanding of disorders, their prevention, and therapy. Proper training for emergency obstetric care is essential. This needs to be followed by placement of human resources with infrastructure and material available. Knowledgeable, skilled persons will become frustrated if infrastructure is not conducive. Understanding precisely where to concentrate efforts, capacity, resources, infrastructure, policies are essential.

Keywords: Disorders during pregnancy, Postpartum to prevent, Severe morbidity, Mortality by appropriate, Emergency obstetric care, Knowledge, skill, Infrastructure, material and policies, Homeostasis, Antibiotics, Appropriate surgery
Volume 8 Issue 2 - 2017

\section{Chhabra S}

Obstetrics Gynecology, Mahatma Gandhi Institute of Medical Sciences, India

Correspondence: Chhabra S, Obstetrics Gynecology, Mahatma Gandhi Institute of Medical Sciences, India, Emailschhabra@mgims.ac.in

Received: February 23, 2017 | Published: November 27, 2017

\section{Introduction}

Prevention of some disorders; which kill many women and babies; is not possible and research continues; but waiting for results of research means many more maternal / neonatal deaths. So the attempts need to be made to try early detection of the disorders during pregnancy; labour and postpartum; prevent severe morbidity \& mortality by evidence-based; timely management. However immediate impact needs quick detection of emergencies and emergency obstetric care (EMOC).

\section{Objectives}

To know about current status of essentialities of emergency obstetric care.

\section{Methodology}

Present article is based on literature search by possible search engines; PubMed; database; update; Google and other database added by own experiences.

\section{Present status}

Recent report by UNICEF revealed that globally maternal deaths decreased from 532000 in 1990 to 303000 in 2015. Likewise; the global maternal mortality ratio (MMR) declined by $44 \%$; from 385 maternal deaths per 100000 live births in 1990 to 216 in 2015; less than half of the 5.5 percent annual decline rate needed. For India MMR declined from 556 in 1990 to 174 in 2015. ${ }^{1 ; 2}$ According to Maternal \& Child Mortality and Total Fertility Rates; Sample Registration System (SRS); National Health Profile report of office of Registrar General of India; the MMR in 2011 was 212; an appreciable decline from 437 in the year 1990; however; far from the goal of National Rural Health Mission (NRHM) India and Mellium Development Goals (MDGs); of less than 109 for $2015 .{ }^{1}$ So for achieving the new goal; it is essential to take steps to accelerate the pace of decline of the MMR in India. The same may be true for many other developing countries. Here comes the role of EMOC.

The International Safe Motherhood Conference convened in Kenya in 1987; raised global awareness of the devastating maternal mortality rates and ratios in developing nations and formally established the 'Safe Motherhood Initiative'. ${ }^{3}$ The goal was to reduce the maternal mortality by $50 \%$ by the year 2000 ; and make the global community aware of the plight of the pregnant women. In the 3 decades; since the discussions about causes of maternal deaths at the International Safe Motherhood Conference and the battle against maternal mortality and morbidity; international public health community has recognized that effective solutions required a coordinated combination of 
knowledge; skills; infrastructure; appropriate technology; policies and their implementation. Implementing public interventions in low-resource settings; where human; financial resources and the physical; epidemiological; environments are constrained; is really challenging. No wonder; only modest progress has been made since the alarm was first sounded. It is essential to have situation analysis; so that prevention can be looked into. Many women suffer because of pregnancy and birth related disorders; quite a few do well; others become severely ill; suffer permanent disabilities and some even die. Maternal deaths are merely the tip of the iceberg of sufferings; because for every woman who dies; many suffer due to various disorders; some recover completely; others live with lifelong disabilities. ${ }^{4}$ Therefore an interest in investigating cases of severe obstetric morbidities; maternal near miss cases; is also essential. Research about MNM continues; as the pathway to MNM and maternal deaths are the same. In most of the situations it is possible to prevent complications; their severity and deaths through appropriate services; especially timely EmOC. However the words are being revised; from obstetric care to essential obstetric care to emergency obstetric care and now to critical obstetric care; but maternal deaths continue to occur. It is essential to go in depth of the reasons and find solutions. Maternal mortality in resource-poor nations has been attributed to the "three delays"; delay in deciding to seek care; delay in reaching care; and delay in receiving appropriate and adequate treatment..$^{5-7}$ There are many sub delays with these three delays.

\section{Disorders which need EMOC}

The list of disorders which could create emergency situations and kill women is long; Abortions; Ectopic Pregnancy; Gestational Trophoblastic Disease; Ante partum Haemorrhage (Placenta Previa and Placental Abruption); Severe Preeclampsia; Eclampsia and HELLP Syndrome; Prolonged Rupture of Membranes with Chorioamnionitis; Complications of Induced labour or Rupture Uterus; Amniotic Fluid Embolism; Third Stage or Immediate Postpartum Complications (Inversion of uterus; Retained placenta; Cervical Vaginal Tears); Postpartum Haemorrhage; Postpartum Shock; Puerperal Sepsis; but actions needed are not many. It is essential to achieve Hemostasis; provide antibiotics provide appropriate surgery and other symptomatic therapy. Abortions; Placenta praevia and PPH lead to vaginal bleeding; Rupture Uterus; Ectopic Pregnancy and Invasive mole might lead to intra uterine or intra peritoneal bleeding and there may not be any vaginal bleeding. Also visible bleeding in some cases could be deceptive. Knowledge of this is essential. Sudden collapse is also known. Woman with septic shock with hyperpyrexia; tachycardia; tachypnoea; evidence of pus intraperitoneal or intra viscera; injury to bladder/bowel; require procedures for which skilled persons; material and infrastructure are essential. Hypertensive disorders; especially complicated by Hepatic; Renal; Cardiovascular Failure; Cerebral Hemorrhage; Unconsciousness; Coma; Disseminated Intravascular Coagulation are dangerous, knowledge to diagnose and manage is essential. $^{8}$

Disorders like cardiomyopathy and acute fatty liver of pregnancy which can occur during pregnancy; labour; post birth need special attention; as the case fatality is high in these cases even when resources are available. ${ }^{9}$ Pregnant women are not immune to other medical/ surgical disorders like different types of hepatitis A; B; C; E; Cerebral malaria; Dengue; Meningitis; Encephalitis; H1N1 viral disease; HIV/ AIDS; Pneumonia; Adult respiratory distress syndrome (ARDS); Pulmonary embolism; Myocardial infarction; Stroke; Cancers; Anaphylaxis to drugs; Surgical emergencies; Burns; Poisoning; Trauma due to Violence; or anaphylaxis following administration of a drug. One has to treat basic disorder and prevent the sequelae; not forgetting the baby. For seeking appropriate timely care; awareness of all possible emergencies is essential.

\section{Emergency services and CS rates}

It is essential that maternal health providers have the knowledge of pregnancy disorders but there are birth related issues too. Skill of $\mathrm{CS}$ at right place and material is essential. Also although the $\mathrm{CB}$ may; at first glance appear to provide a relatively safe and certain outcome; it increases morbidity/mortality. ${ }^{10-12}$ It is essential that the health providers understand this also. It is essential to view the widely increasing rates of CS; as potential obstetric hazard. CS when medically indicated and performed by trained staff can be a lifesaving procedure for the mother and baby. But compared to vaginal delivery, CS is associated with a higher risk of maternal and neonatal death; maternal morbidity including infection and amniotic fluid embolism; neonatal morbidity often related to iatrogenic prematurity; and future uterine rupture potential, other complications in subsequent pregnancies. Studies have also revealed that children born via CS are more likely to develop respiratory problems; diabetes and obesity later in life. Researchers have debated the optimal CS rate for maximizing maternal and infant health outcomes. ${ }^{13}$ World Health Organization suggested the ideal CSR of $10-15 \%{ }^{14}$ although some scientists have suggested revisit of earlier rates. ${ }^{15,16}$ Further investigations of an optimal rate are certainly warranted. In 1990; roughly one in 15 babies on the planet was born via CS and in 2014; one in five babies was born via CS. ${ }^{13}$ There are wide geographic variations in CSR; but almost all show a rise; quadrupled in less than two decades; even in two nearby countries; Holland and Denmark have rates of $6.5 \%$ and $12.1 \%$ respectively. ${ }^{17}$ Variations in CS rates among lowrisk women illustrated that a woman's health status alone did not account for the trend. Convenience factors and the threat of medical malpractice lawsuits created incentives for providers to choose CS over vaginal delivery. Evidence suggested that higher CS rate can be associated with worse outcomes. ${ }^{15,18-20}$

The use of CS has increased to unprecedented levels worldwide although the gap between higher and lower resource settings remains. ${ }^{21-26}$ This increase deserves international attention; not only because of health consequences; resource administration and policies; but also because high CSRs are not associated with low perinatal mortality rates. Despite the fact that the $\mathrm{WHO}^{27}$ has reported that no region in the world is justified in having a CSR greater than 10 to 15 percent; the rates have been escalating. However Molina et $\mathrm{al}^{28}$ reported CSR upto approximately 19 per 100 live births associated with lower maternal or neonatal mortality among WHO member states and suggested that previously recommended national target rates for CB may be too low. By 1999 the CSR in UK and USA had reached 20 $\%$ and $22 \%$ respectively. ${ }^{29,30} \mathrm{~A}$ tertiary care unit in Sri Lanka reported an increase in the CSR from $13 \%$ in 1985 to $22 \%$ in $1999 .{ }^{31}$ However Ye et al. ${ }^{32}$ have suggested that; at the population level; CSR higher than around $10 \%$ were not associated with substantial decrease in maternal and neonatal mortality; after controlling for socio economic conditions. Based on the available ecological evidence; higher CSR may not be necessary to achieve the lowest maternal and neonatal mortality rates at the population level. Actually the PMR was found to increase as a consequence of trying to improve the perinatal outcome of the very low-birth-weight babies by a liberal CS policy. ${ }^{33}$ A U.S. population-based study of neonatal and infant mortality by mode of delivery among women with "no indicated risk;" showed that neonatal mortality increased more than two-fold after CB; even after excluding infants with congenital anomalies and presumed intrapartum hypoxic 
events and adjusting for demographic and medical covariates..$^{34}$ Yip et al. ${ }^{35}$ reported that CS was significantly associated with risk of autism spectrum disorder for each week of gestation; from week 36 to 42; consistently across study sites. A large increase in CS; many fruitless; has been found in a study in rural eastern part of Maharashtra; India. ${ }^{36}$ The precise relationship between increasing CSR and maternal; perinatal mortality (PM) \& morbidity is not very well understood. Tendency to regard CS as an uncomplicated procedure in the affluent world has increased its incidence in developing world also; overlooking the complications that can occur in anemic; malnourished women. Further the risk of perinatal mortality and morbidity; maternal morbidity; mortality believed to increase when CS is performed as an emergency procedure after prolonged labour. ${ }^{36}$ This needs to be part of EMOC training too. It is essential that while providing knowledge and skills of EMOC; health providers are made aware of this aspect of health care too. ${ }^{11}$

As such poor quality EMOC is a major concern, not only because it is a major cause of maternal deaths and disabilities; but because it can lead to the underutilization of maternal health services. ${ }^{37,38}$ Geographic location with its milieu and health services; are also key determinant of quality of EMOC as indicated by studies that revealed major differentials in the quality of maternal health offered in different parts. Whatever may be the scenario; quality has to be in place on a continuous basis. ${ }^{39}$

\section{Possibilities}

Focus on interventions that are designed to avert deaths and disabilities to women during pregnancy; or delivery or in the immediate postpartum period; is essential; Advocating a single universal approach of implementation does not help. Neither is it possible that every situation is so unique that it has to start from scratch. Emphasis on competency; rather than simply knowledge in a core set of essential skills; ensuring that skilled manpower fits into the infrastructural realities of high-mortality; low-resource settings; is imperative. ${ }^{40-42}$

Women; communities; health professionals need to be aware. A system of birth preparedness and complications readiness amongst the women and the communities need to be in place. Maternal mortality reduction in severely sick needs appropriately skilled human resources and the infrastructure to treat life-threatening complications. There has to be awareness that some sick women need intensive care; sometimes cardio respiratory support; mechanical ventilation; cardio tonics and massive blood/components transfusion. Life saving measures become essential; may be obstetric hysterectomy; internal iliac ligation etc. or simple evacuation of uterine cavity or suturing cervical tear. So timely appropriate referral is essential and skilled manpower; specialists or trained non-specialists knowing; their job as per the need; comprehensive understanding of the disorders and emergency care needed; knowledge; skills and material. Non specialist can be trained to provide the needed services. ${ }^{43}$

Proper training; proper placement of human resources and infrastructure available; are all essential for EMOC. Policy makers and programme managers must ensure that all the three are in place. Policy makers need to look into ground realities of infrastructure; possible low cost technologies; be it use of partograph or active management of third stage of labour; which prevents third stage postpartum complications; including $\mathrm{PPH} .{ }^{44}$ Obstructed labor is an important cause of maternal and perinatal mortality and morbidity. ${ }^{45}$ The partograph graphically represents key events in labor and provides an early warning system; prevents prolonged labour and obstructed labour. However; challenges in implementation continue and need to be addressed urgently ${ }^{46}$ and research needs to continue to find simple modes which emergency staff find easy to use and prevent complications. Further wonderfully knowledgeable; skilled persons will become frustrated if infrastructure is not conducive. The disorders and adverse events known to lead to maternal deaths are known globally; prevention of disorders may or may not be possible but prevention of deaths and disabilities is possible by EMOC; by having appropriately trained persons who take appropriate actions; placed at appropriate places which have infrastructure. There is no point in training if proper placement cannot be done. What is needed is to know; as to who has to do what; from government to community and persons; community centre to referral. Even with resource constraints; mortality due to disorders like Eclampsia; obstetric hemorrhage can be reduced with appropriate and timely therapy. Evidence-based critical care is essential as even seemingly milder forms of disorders if underestimated can lead to mortality. ${ }^{47}$

For all these actions right persons are needed. If specialists are not available non specialists can have the comprehensive training/ learning. Knowledge \& skill to do a CS; while needs to be part of essentialities of emergency services; is not the panacea of prevention of morbidity and mortality; as women are not dying; because CS is not available; but also because disorders like hypertension; sepsis; PPH are not prevented; not diagnosed in time, not treated appropriately and timely. Although indicated and timely CS is of tremendous benefit to the mother and the baby; the repercussions; sequelae and health economics demand a better understanding of the situation and this aspect needs to be hailed during EMOC training. While it is essential to train as to how to do CS; it is also essential to train decision making \& therapy of other disorders. Also training for forceps; evacuation; vacuum extraction; suturing of tears and treatment of fatal disorders like Eclampsia; abruption is essential. It is essential to understand the delays and sub delays; if solutions are to be found. Analysis of various factors can identify bottlenecks; which contribute to maternal deaths at various levels. The information can be used to take steps to fill the gaps in the services. It is essential that the maternal health providers have knowledge; skills and at the places where maternal health care is provided; there is infrastructure and material. For all this to happen; right programs and policies are must. Programs aimed at strengthening capacity to deliver emergency obstetric services in health facilities have been started to integrate skills; knowledge with guidelines and plans of basic equipments and supplies. With high risk approach and timely referral; community based basic care; birth preparedness and complications awareness; timely referral to appropriate facility; which has back up of managing eventualities; is the hallmark of saving lives. Let the drops of training become an ocean which is needed to help the global mothers \& their tiny tots through EMOC.

\section{Acknowledgments}

None.

\section{Conflicts of Interest}

No conflict of interest.

\section{References}

1. Maternal mortality fell by almost half between 1990 and 2015. 2017

2. Iversen K. Diabetes in pregnancy-a neglected cause of maternal mortality. 2007. 
3. Nour NM. An Introduction to Maternal Mortality Rev Obstet Gynecol. 2008;1(2):77-81.

4. Koblinsky MA. Beyond maternal mortality-magnitude, interrelationship, and consequences of women's health, pregnancyrelated complications and nutritional status on pregnancy outcomes. Int J Gynaecol Obstet. 1995;48(Suppl):S21-S32.

5. Maine D. Safe Motherhood Programs: Options and Issues. Center for Population and Family Health, Columbia University, New York, USA. 1991.

6. Maternal Mortality Update 2002: A Focus on Emergency Obstetric Care. UNFPA, USA. 2003.

7. Bakshi. Report of the Special Rapporteur on the right of everyone to the enjoyment of the highest attainable standard of physical and mental health. 2015.

8. Kezić A, Sparić R, Stojimirović B, et al. Multiorgan dysfunction in a gravid woman with placental abruption and disseminated intravascular coagulation. Srp Arh Celok Lek. 2007;135(7-8):465-467.

9. Keyser EA, Reed BG, Gonzalez-Brown V, et al. Peripartum cardiomyopathy and acute fatty liver of pregnancy: one patient with two zebras. Mil Med. 2007;177(4):470-473.

10. Srp B, Velebil P. Proportion of cesarean sections and main causes of maternal mortality during 1978-1997. Ceska Gynekol. 1999;64(4):219223.

11. Lydon-Rochelle M, Holt VL, Martin DP, et al. Association between method of delivery and maternal rehospitalization. JAMM. 2000;283(18):2411-2416.

12. Chaim W, Bashri A, Bar-David J, et al. Prevalence and clinical significance of postpartum endometritis and wound infection. Infect Dis Obstet Gynecol. 20008(2):77-82.

13. Hodin S. The Global Epidemic of Unnecessary Cesarean Sections (Part 2). 2017.

14. Mukherjee SN. Rising cesarean section rate. J Obstet Gynecol India. 2006;56(4):298-300.

15. Victora CG, Barros FC. Beware: unnecessary caesarean sections may be hazardous. Lancet. 2006;367(9525):1796-1797.

16. What is the right number of caesarean sections? Lancet. 1997;349(9064):815-816.

17. Savage W, Francome C. British caesarean section rates: have we reached a plateau? Br J Obstet Gynaecol. 1993100(5):493-496.

18. Steer PJ, Modi N. Elective caesarean sections-risks to the infant Lancet. 2009;374(9691):675-676.

19. Caesarean section-the first cut isn't the deepest. Lancet. 2010;375(9719):956.

20. Mi J, Liu F. Rate of caesarean section is alarming in China. Lancet. 2014;383(9927):1463-1464

21. Murray SF, Serani-Pradenas F. Cesarean birth trends in Chile, 19861994. Birth. 1997;24(4):258-263.

22. Bulger T, Howden-Chapman P, Stone P. A cut above: the rising cesarean section rate in New Zealand. Med. 1998;J11:30-33.

23. Flamm B1, Berwick DM, Kabcenell A. Reducing cesarean section rates safely: Lessons from a breakthrough series collaborative. Birth. 1998;25(2):117-124.

24. Wu W1. Cesarean delivery in Shantou, China: a retrospective analysis of 1922 women. Birth. 2000;27(2):86-90.

25. Sheiner E, Shohan-VardiI, Hershkovitz R, Kazt M, et al. Infertility treatment is an independent risk factor for cesarean section among mulliparous women aged 40 and above. Am J Obstet Gynecol. 2001;185(4):888-892.

26. Betrán AP, Ye J, Moller AB, et al. The Increasing Trend in Caesarean Section Rates: Global, Regional and National Estimates:. 2016;19902014;5:11(2)

27. Yazdizadeh B, Nedjat S, Mohammad K, et al. Caeserian section rate in Iran, multidimensional approaches for behavioural change of providers: a quantitative study. BMC Health Serv. Res. 2011 ;(11):159.

28. Molina G, Weiser TG, Lipsitz SR, et al. Relationship Between Cesarean Delivery Rate and Maternal and Neonatal Mortality. JAMA. 2015;314(21):2263-2270.

29. Cesarean section is on the rise. Lancet. 2000;356:1997-1998.

30. Ferriman A. Cesarean section rate hits $20 \%$ in 1999-2000. British Medical Journal. 2001;322:1508-1510.

31. Gunarathna KA, Goonewardane IMR. The rise in cesarean section rates. Srilanka Journal Obstetrics Gynaecology. 2001;46(4):147-150.

32. Ye J, Zhang J, Mikolajczyk R, et al. Association between rates of caesarean section and maternal and neonatal mortality in the 21 st century: a worldwide population-based ecological study with longitudinal data. BJOG. 2015;123(5):745-753.

33. Signore C, Klebanoff M. Neonatal Morbidity and Mortality After Elective Cesarean Delivery. Clin Perinatol. 2008;35(2):361-371.

34. MacDorman MF, Declercq E, Menacker F, et al. Infant and neonatal mortality for primary cesarean and vaginal births to women with "no indicated risk," United States, 1998-2001 birth cohorts. Birth. 2006;33(3):175-182.

35. Yip BHK, Leonard H, Stock S, et al. Caesarean section and risk of autism across gestational age: a multi-national cohort study of 5 million births. Int J Epidemiol. 2016.

36. Warade S, Gore S, Zawar G. Caesarean section for non progress of labour and perinatal outcome. International Journal of Recent Trends in Science and Technology. 2016;19(3):377-380.

37. Leduc D, Senikas V, Lalonde AB, et al. Active management of the third stage of labour: prevention and treatment of postpartum hemorrhage. $J$ Obstet Gynaecol Can. 2009;31(10):980-993.

38. McGraw D, Greene SM, Miner CS, et al. Privacy and confidentiality in pragmatic. Clin Trials. 2016;12(5):520-529.

39. Nair S. Visual Merchandising and Retail design. 2011;6:25.

40. AMDD. Emergency obstetric care: resources and tools CD-ROM. Baltimore: JHPIEGO. 2003.

41. Islam MT, Haque YA, Waxman R, et al. Implementation of emergency obstetric care training in Bangladesh: lessons learned. Reprod Health Matters. 200614(27):61-72.

42. Makowiecka K, Achadi E, Izati Y, et al. Midwifery provision in two districts in Indonesia: how well are the rural villages served? Health Policy Plan. 2008;23(1):67-75.

43. Das J, Hammer J, Leonard K. J Econ Perspect. 2008;22(2):93-114.

44. Assessment of Training of Medical Officers in Emergency Obstetric Care Including Caesarean under Nrhm/Rch-Ii.

45. Indra , Usharani N, Mumtaz Bendigeri. Global burden of obstructed labour. Int J Reprod Contracept Obstet Gynecol. 2000;6(2):439-442.

46. Mathai M. The partograph for prevention of obstructed labor: Clin Obstet Gynaecol . 2009;52(2):256-269.

47. Chhabra S, Jaiswal A, Raman S. Persistence of fruitless caesarean section in Indian rural women. J Obstet Gynaecol. 2009;29(8):718-721. 